\title{
Outcomes in patients undergoing coronary artery bypass graft surgery in the United States based on hospital volume, 2007 to 2011
}

\author{
Luke K. Kim, MD, ${ }^{\text {a }}$ Patrick Looser, MD, ${ }^{\text {a }}$ Rajesh V. Swaminathan, MD, ${ }^{\text {a }}$ Robert M. Minutello, MD, ${ }^{a}$ \\ S. Chiu Wong, MD, ${ }^{a}$ Leonard Girardi, MD, ${ }^{b}$ and Dmitriy N. Feldman, MD ${ }^{a}$
}

\section{ABSTRACT}

Objective: To examine national trends in coronary artery bypass grafting (CABG) volume between 2007 and 2011, and analyze in-hospital outcomes after CABG surgery stratified according to hospital volume.

Methods: We analyzed all patients who underwent isolated CABG surgery between 2007 and 2011 in the National Inpatient Sample database. Trends in procedure volume and rates of adverse in-hospital outcomes were examined. Multivariate propensity-score adjusted analysis was performed to compare in-hospital mortality for hospitals based on quartiles of CABG volume.

Results: The frequency of isolated CABG decreased by $25.4 \%$ from 2007 to 2011 (from 326 cases per million adults to 243 cases per million adults), with the most marked decline at higher-volume centers. Patients in the highest-volume quartile were more likely to have a history of previous $\mathrm{CABG}$, previous percutaneous coronary intervention, peripheral vascular disease, hypertension, or chronic renal failure. In-hospital mortality was highest in low-volume centers. In multivariate logistic regression analysis, low hospital volume was an independent predictor of in-hospital all-cause mortality (adjusted odds ratio, 1.39; $95 \%$ confidence interval, 1.24-1.56; $P<.001)$.

Conclusions: The rate of CABG procedures has declined, mainly at high-volume centers. Low CABG volume is associated with an increase in in-hospital mortality. (J Thorac Cardiovasc Surg 2016;151:1686-92)

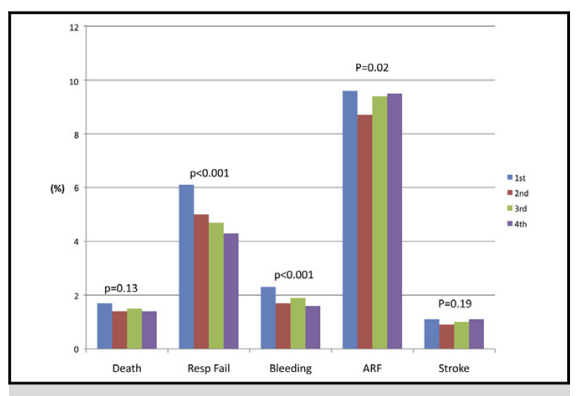

Adverse event rates after elective CABG by quartiles of hospital volume.

\section{Central Message}

Hospital volume is a predictor of procedural outcomes after CABG. Lower volume centers tend to have higher in-hospital mortality.

\section{Perspective}

CABG volume continues to decline with improved medical therapy and PCI for coronary artery disease. Low hospital volume correlates with worse postsurgical outcomes in our study. Hospital CABG volume should be taken into account when delivering care to patients with ischemic heart disease.

See Editorial Commentary page 1693.
Previous studies have demonstrated a clear relationship between volume and outcomes for various surgical and interventional procedures. Higher-volume centers have been shown to have improved clinical outcomes over

From the ${ }^{\mathrm{a} D i v i s i o n}$ of Cardiology, Department of Medicine and ${ }^{\mathrm{b}}$ Department of Cardiothoracic Surgery, Weill Cornell Medical College, New York Presbyterian Hospital, New York, NY

This work was supported by grants from the Michael Wolk Heart Foundation and the New York Cardiac Center, Inc. The Michael Wolk Heart Foundation and the New York Cardiac Center, Inc. had no role in the design and conduct of the study, in the collection, analysis, and interpretation of the data, or in the preparation, review, or approval of the manuscript.

L.K.K. and D.N.F. take responsibility for all aspects of the reliability and freedom from bias of the data presented and their discussed interpretation.

Received for publication Sept 21, 2015; revisions received Jan 11, 2016; accepted for publication Jan 26, 2016; available ahead of print March 8, 2016.

Address for reprints: Luke K. Kim, MD, Division of Cardiology, Department of Medicine, Weill Cornell Medical College/New York Presbyterian Hospital Starr Pavilion, 4th Floor, 520 East 70th St, New York, NY 10021 (E-mail: luk9003@ med.cornell.edu).

$0022-5223 / \$ 36.00$

Copyright (c) 2016 by The American Association for Thoracic Surgery

http://dx.doi.org/10.1016/j.jtcvs.2016.01.050 low-volume institutions in a variety of cardiovascular surgical procedures, including carotid endarterectomy, heart transplantation, acute aortic dissection repair, percutaneous coronary intervention, and valve surgery. ${ }^{1-4}$ Particularly for percutaneous coronary intervention (PCI), there is a significant inverse correlation between PCI volume and outcomes. ${ }^{4}$ Data on coronary artery bypass grafting (CABG) are conflicting, however, with several studies demonstrating improved outcomes at highervolume centers with experienced operators ${ }^{5-8}$ but other studies failing to confirm these findings. ${ }^{9}$

With the changing landscape of coronary revascularization, it is important to evaluate the relationship between

Scanning this QR code will take you to the article title page. 


\section{Abbreviations and Acronyms \\ $\mathrm{ARF} \quad=$ acute renal failure \\ CABG = coronary artery bypass grafting \\ $\mathrm{CAD}=$ coronary artery disease \\ ICD-9-CM = International Classification of \\ Diseases, Ninth Revision, Clinical \\ Modification \\ NIS = Nationwide Inpatient Sample \\ PCI = percutaneous coronary intervention}

declining CABG volume and patient outcomes. Given the paucity of recent data on CABG volume and postsurgical outcomes, we sought to examine national trends in CABG volume between 2007 and 2011. Importantly, we analyzed in-hospital outcomes after CABG surgery stratified according to hospital volume.

\section{METHODS}

Data were obtained from the Agency for Healthcare Research and Quality's Healthcare Cost and Utilization Project, Nationwide Inpatient Sample (NIS) files between 2007 and $2011 .{ }^{10}$ The NIS is a 20\% stratified sample of all nonfederal US hospitals. In 2011, the NIS contained deidentified information for 38,590,733 discharges from 1049 hospitals and 46 states. Discharges are weighted based on the sampling scheme to permit inferences for a nationally representative population. ${ }^{10}$ Each record in the NIS includes all procedure and diagnosis International Classification of Diseases, Ninth Revision, Clinical Modification (ICD) codes recorded for each patient's hospital discharge.

Hospitalizations leading to CABG between January 2007 and December 2011 were selected by searching for the ICD-9-CM procedure codes $36.10,36.11,36.13,36.14,36.15,36.16,36.17$, and 36.19 in any of the 15 procedure fields in the database. Off- pump CABG surgery was identified using ICD-9-CM codes 39.61 and 39.62. Concomitant valve surgeries were identified by ICD-9-CM code 35.2. Patients with stable coronary artery disease (CAD) were selected using the algorithm of Mohan et al. ${ }^{11}$

Patient-level and hospital-level variables were included as baseline characteristics. Hospital-level data elements were derived from the AHA Annual Survey Database. The Agency for Healthcare Research and Quality's comorbidity measures based on the Elixhauser method were used to identify comorbid conditions. ${ }^{12}$ The primary outcome was in-hospital all-cause mortality for the overall cohort of isolated CABG. Secondary outcome measures were in-hospital all-cause mortality, stroke, bleeding, respiratory failure, and acute renal failure (ARF) for a cohort of elective, isolated CABG procedures. Stroke was identified by ICD-9-CM codes 997.02, 362.31, 368.12, 781.4, 433.11, 435, and 434. Respiratory failure was identified by ICD-9-CM codes 518.81, 518.84, and 799.1. Major bleeding was identified by ICD-9-CM codes 430 to 432, 578.X, 719.1X, 423.0, 599.7, 626.2, 626.6, 626.8, 627.0, 627.1, 786.3, 784.7, and 459.0. ARF was identified by ICD-9-CM code 584

The procedure rates was calculated as the weighted number of isolated CABG procedures divided by $20 \%$ of the total number of US adults during the same period. Estimates of the US adult population for 2007 to 2011 were obtained from the US Census Bureau. ${ }^{13}$ Trends in the annual rates of isolated CABG for elective and nonelective indications were assessed using time series modeling. Rates of CABG over time were compared in subgroups based on hospital volume status. Hospital volume status was defined by quartiles based on the volume of CABG performed between 2007 and 2011. The first quartile was defined as centers with the lowest
CABG volume. In addition, the rates of CABG were analyzed for subgroups based on pump utilization (off-pump vs on-pump surgery).

For descriptive analyses, we compared baseline patient characteristics and hospital characteristics by quartile of hospital volume. Continuous variables are presented as medians; categorical variables, as frequencies (percentages). Baseline characteristics and in-hospital care patterns with respect to hospital volume were compared using either the Mann-Whitney-Wilcoxon nonparametric test or Student $t$ test for continuous variables and the Pearson $\chi^{2}$ test for categorical variables. All statistical tests were 2 -sided, and a $P$ value $<.05$ was set a priori to be statistically significant.

Unadjusted in-hospital mortality rates were calculated according to the isolated CABG (ie, excluding concomitant valve surgery) volume status. Multivariable logistic regression analyses were used to compare outcomes between each quartile (with the fourth quartile as the reference) adjusting for univariate predictors of outcomes $(P<.01)$ from among left internal mammary artery used, bilateral internal mammary arteries used, off-pump bypass surgery, renal dialysis, previous CABG, previous PCI, age, sex, race, hospital bed size, hospital teaching status, region, payer, anemia, collagen vascular disease, congestive heart failure, chronic pulmonary disease, pulmonary circulation disorder, diabetes mellitus, coagulopathy, hypertension, liver disease, neurologic disorders, obesity, stable CAD, peripheral vascular disorders, renal failure, and valvular disease. For all regression analyses, the Taylor linearization method "with replacement" design was used to compute variances. To study a low-risk stable $\mathrm{CAD}$ population undergoing $\mathrm{CABG}$, a separate multivariate logistic regression analysis was performed for evaluation of elective hospitalizations leading to isolated CABG surgeries. All statistical tests were 2 -sided, and a $P$ value $<.05$ was set a priori to be statistically significant. All multivariate regression analyses were performed using SAS version 9.2 (SAS Institute, Cary, NC) and SPSS version 20 (IBM, Armonk, NY).

\section{RESULTS}

For each year from 2007 to 2011, the NIS dataset included discharges from all hospitals that performed CABG, which increased from 240 hospitals in 2007 to 245 hospitals in 2011. Of 196,461,055 discharge records reviewed between 2007 and 2011, 207,441 patients underwent isolated CABG, of which $46.3 \%$ were elective admissions. Table 1 compares baseline characteristics stratified by hospital volume (in quartiles). Patients undergoing CABG performed in highest-volume versus lowest-volume centers (fourth quartile vs first quartile) were more likely to have a history of previous CABG $(1.5 \%$ vs $1.1 \% ; P<.001)$, previous PCI $(12.8 \%$ vs $12.0 \% ; P=.02)$, peripheral vascular disease $(15.0 \%$ vs $14.3 \% ; P<.001)$, chronic renal failure $(13.0 \%$ vs $12.4 \% ; P=.003)$, and hypertension $(75.4 \%$ vs $73.8 \%$; $P<.001)$.

The annual rate of isolated CABG surgeries decreased by $25.4 \%$ ( $P$ for trend $=.08$ ) from 325.8 procedures per million adults per year in 2007-2008 to 242.9 procedures per million adults per year in 2010-2011 (Table 2). The median number of cases in the 4 quartiles (lowest to highest) in 2007 was 47, 117, 185, and 334. In the year 2011, the median number of cases in these quartiles was 48, 93, 172, and 296. Although the decline in CABG volume was not statistically significant in the lowest 
TABLE 1. Baseline and hospital characteristics of patients undergoing isolated CABG in 2007 to 2011 by hospital volume in quartiles

\begin{tabular}{|c|c|c|c|c|c|}
\hline Characteristic & $\begin{array}{c}\text { First quartile } \\
(n=13,311)\end{array}$ & $\begin{array}{l}\text { Second quartile } \\
\quad(\mathbf{n}=\mathbf{3 3 , 3 8 0})\end{array}$ & $\begin{array}{c}\text { Third quartile } \\
(n=49,008)\end{array}$ & $\begin{array}{c}\text { Fourth quartile } \\
(\mathbf{n}=\mathbf{1 1 1 , 7 4 2 )}\end{array}$ & $P$ value \\
\hline Age, year, mean \pm SD & $64.6 \pm 10.9$ & $65.1 \pm 10.7$ & $65.2 \pm 10.6$ & $65.1 \pm 10.8$ & $<.001$ \\
\hline Female sex, $\%$ & 27.5 & 26.9 & 26.9 & 27.4 & .12 \\
\hline Hypertension, \% & 73.8 & 74.3 & 75.1 & 75.4 & $<.001$ \\
\hline Diabetes mellitus, uncomplicated, $\%$ & 34.5 & 34.4 & 33.2 & 32.7 & $<.001$ \\
\hline Diabetes mellitus, complicated, \% & 6.8 & 6.5 & 6.2 & 6.9 & $<.001$ \\
\hline Chronic renal failure, $\%$ & 12.4 & 12.4 & 12.5 & 13.0 & .003 \\
\hline Congestive heart failure, $\%$ & 1.2 & 1.0 & 0.9 & 1.0 & .02 \\
\hline Chronic pulmonary disease, $\%$ & 22.1 & 21.5 & 23.3 & 21.8 & $<.001$ \\
\hline Obesity, $\%$ & 16.9 & 17.0 & 18.2 & 18.2 & $<.001$ \\
\hline Coagulopathy, \% & 11.8 & 11.4 & 13.0 & 11.7 & $<.001$ \\
\hline Neurologic disorder, $\%$ & 3.2 & 3.2 & 3.2 & 3.2 & .92 \\
\hline Anemia, $\%$ & 23.4 & 19.9 & 21.2 & 17.3 & $<.001$ \\
\hline Collagen vascular disease, $\%$ & 1.5 & 1.7 & 1.7 & 1.7 & .23 \\
\hline Peripheral vascular disease, $\%$ & 14.3 & 13.9 & 15.2 & 15.0 & $<.001$ \\
\hline Liver disease, $\%$ & 1.1 & 1.2 & 1.1 & 1.0 & .14 \\
\hline Fluid and electrolyte imbalance, $\%$ & 22.2 & 21.8 & 22.7 & 24.4 & $<.001$ \\
\hline Pulmonary circulation disorder, $\%$ & 0.2 & 0.2 & 0.2 & 0.2 & .79 \\
\hline Previous CABG, $\%$ & 1.1 & 1.1 & 1.3 & 1.5 & $<.001$ \\
\hline Previous PCI, \% & 12.0 & 12.1 & 12.7 & 12.8 & .02 \\
\hline LIMA used, $\%$ & 85.2 & 85.6 & 86.0 & 85.0 & $<.001$ \\
\hline BIMA used, \% & 2.9 & 3.5 & 3.3 & 4.7 & $<.001$ \\
\hline Off-pump CABG, $\%$ & 24.8 & 28.7 & 24.5 & 25.9 & $<.001$ \\
\hline Elective, $\%$ & 46.7 & 47.1 & 48.3 & 45.1 & $<.001$ \\
\hline Teaching hospital, \% & 32.2 & 46.2 & 45.4 & 69.5 & $<.001$ \\
\hline Urban, $\%$ & 87.3 & 90.0 & 92.6 & 98.0 & $<.001$ \\
\hline Race, \% & & & & & $<.001$ \\
\hline White & 68.0 & 71.2 & 80.3 & 81.8 & \\
\hline Black & 8.3 & 6.6 & 6.1 & 7.6 & \\
\hline Hispanic & 12.1 & 12.5 & 7.1 & 4.9 & \\
\hline Hospital bed size, $\%$ & & & & & $<.001$ \\
\hline Small & 12.1 & 12.5 & 7.6 & 6.2 & \\
\hline Medium & 34.2 & 26.9 & 18.3 & 13.3 & \\
\hline Large & 53.8 & 60.6 & 74.1 & 80.5 & \\
\hline Hospital region, \% & & & & & $<.001$ \\
\hline Northeast & 4.0 & 12.5 & 8.7 & 19.7 & \\
\hline Midwest & 29.0 & 35.9 & 24.6 & 21.3 & \\
\hline South & 39.0 & 28.3 & 40.3 & 50.5 & \\
\hline West & 28.0 & 23.3 & 26.3 & 8.6 & \\
\hline Length of stay, days, mean \pm SD & $9.8 \pm 7.01$ & $9.4 \pm 7.2$ & $9.4 \pm 7.2$ & $9.5 \pm 7.8$ & $<.001$ \\
\hline Median hospitalization charge, $\$$ & 139,221 & 116,347 & 106,276 & 95,892 & $<.001$ \\
\hline
\end{tabular}

Obesity and anemia are based on the International Classification of Diseases, Ninth Revision, Clinical Modification coding algorithms for Elixhauser comorbidities. There were 62 hospitals in each quartile in 2007. SD, Standard deviation; CABG, coronary artery bypass graft surgery; PCI, percutaneous coronary interventions; LIMA, left internal mammary artery; BIMA, bilateral internal mammary arteries.

quartile, the $\mathrm{CABG}$ rate declined substantially in the second and third quartiles (by $21.1 \%[P=.05]$ and by $48.6 \%$ $[P=.03]$, respectively), with statistically nonsignificant decline in the fourth quartile (Table 2). Given the $2.1 \%$ increase in the number of hospitals performing CABG from 2007 to 2011, the decline in CABG rate reflects a $19.6 \%$ reduction in the median caseload, from 148 cases per hospital in 2007 to 119 cases per hospital in 2011. Figure 1 shows the rates of isolated CABG in key subgroups based on elective versus nonelective admission and on-pump versus off-pump. There was a $25.3 \%$ decrease in the rate of CABG performed from 2007 to 2011 in the nonelective $\mathrm{CABG}$ group ( $P$ for trend $=.05$ ).

The unadjusted incidences of in-hospital death $(2.5 \%$ vs $2.1 \%$ vs $2.1 \%$ vs $2.0 \%$, from lowest to highest quartile of volume; $P=.003$ ) were higher in lower-volume centers (Figure 2). Among subgroups of patients who underwent CABG electively, the unadjusted incidences of respiratory failure $(6.1 \%$ vs $5.0 \%$ vs $4.7 \%$ vs $4.3 \% ; P<.001)$ and bleeding complications $(2.3 \%$ vs $1.7 \%$ vs $1.9 \%$ vs $1.6 \%$; 
TABLE 2. Rates of isolated CABG per million adults by hospital volume in quartile

\begin{tabular}{lrrrrrrr}
\hline Quartile & $\mathbf{2 0 0 7}$ & $\mathbf{2 0 0 8}$ & $\mathbf{2 0 0 9}$ & $\mathbf{2 0 1 0}$ & $\mathbf{2 0 1 1}$ & $\boldsymbol{P}$ value* & \% change $\dagger$ \\
\hline First & 17.8 & 22.3 & 19.1 & 18.1 & 17.7 & .79 & -1.1 \\
Second & 51.3 & 53.1 & 46.8 & 45.8 & 41.0 & .05 & -21.1 \\
Third & 86.3 & 86.2 & 79.5 & 53.7 & 44.4 & .03 & -48.6 \\
Fourth & 170.3 & 175.3 & 180.0 & 130.3 & 139.9 & .23 & -17.9 \\
Total & 325.8 & 336.9 & 325.4 & 247.9 & 242.9 & .08 & -25.4 \\
\hline$* P$ valu for
\end{tabular}

$* P$ value for trend. $\dagger$ Change in volume from 2007 to 2011.

$P<.001$ ) were higher in lower-volume centers (Figure 3). Being a low-volume CABG center (in the first vs fourth quartile of volume) was associated with significantly greater odds of death in both univariate and multivariate logistic regression analyses (Table 3). To further evaluate outcomes in the low-risk CABG cohort $(46.3 \%$ of the study group), when patients were admitted for elective surgery, we performed a multivariate analysis comparing those in lower quartiles of CABG volume with those in the fourth quartile (Table 4). Undergoing elective, isolated CABG in low-volume centers (in the first quartile vs fourth quartile of volume) was independently associated with greater odds of death (adjusted odds ratio $[\mathrm{OR}], 1.44 ; 95 \%$ confidence interval $[\mathrm{CI}], 1.20-1.74$; $P<.001$ ), respiratory failure (adjusted OR, $1.40 ; 95 \% \mathrm{CI}$, 1.24-1.59; $P<.001$ ), and bleeding events (adjusted OR, 1.35; 95\% CI, 1.11-1.64; $P=.002$ ). Table 5 lists independent predictors of in-hospital mortality after correcting for baseline differences with multivariate logistic regression analysis. In fact, after adjusted multivariate analysis, low hospital CABG volume (ie, being in the first quartile vs other quartiles) was an independent predictor of in-hospital all-cause mortality (adjusted OR, 1.34; 95\% CI, 1.17-1.52; $P<.001)$ after isolated CABG surgery.

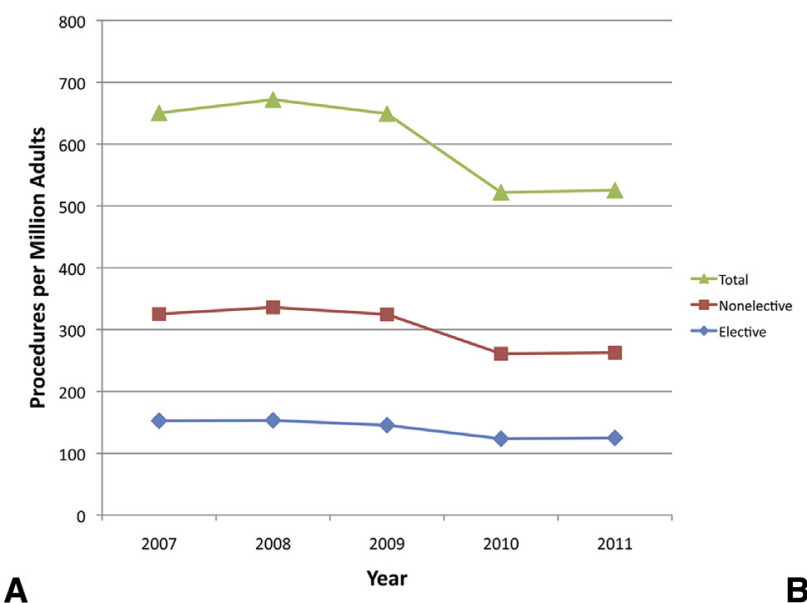

\section{DISCUSSION}

There are several important findings in this nationally representative database of US hospital discharge records analyzing the rates of CABG and outcomes relative to hospital volume between 2007 and 2011. First, the overall CABG rate decreased by $23.1 \%$ from 2007 to 2011 . This decrease was driven primarily by a reduction in the number of cases performed in higher-volume centers. Second, undergoing isolated $\mathrm{CABG}$ in the lowest-volume centers (those in the first quartile of hospital volume) was associated with worse postprocedural all-cause mortality. Undergoing elective CABG in the lowest-volume centers was associated with higher in-hospital mortality, respiratory failure, and bleeding complications. Finally, CABG at low-volume centers was an independent predictor of in-hospital all-cause mortality.

Our findings are consistent with previous reports evaluating utilization of CABG in the United States. ${ }^{14}$ A previous study using NIS data demonstrated a decline in CABG volume by $38 \%$ from 2001 to $2007 .{ }^{15}$ Our more contemporary data demonstrate a continued decline in CABG volume, with further reduction in the volume of CABG by $17.9 \%$ from 2008 to 2011 . There are several plausible explanations for this decline in CABG volume during the study period. It has been clearly demonstrated that improvements in medical therapy as well as lifestyle modification have resulted in reduced rates of CAD and complications of CAD in recent years. ${ }^{16}$ Furthermore, evolution of PCI techniques, low rates of restenosis and stent thrombosis with second-generation drug-eluting stents, low rates of periprocedural complications with PCI have led to an overall increase in the use of PCI and, as a result, may have caused a proportional decrease in CABG utilization. ${ }^{17}$ In addition, it is important to recognize the potential contribution of the economic decline in the United States during the years of our study period, which

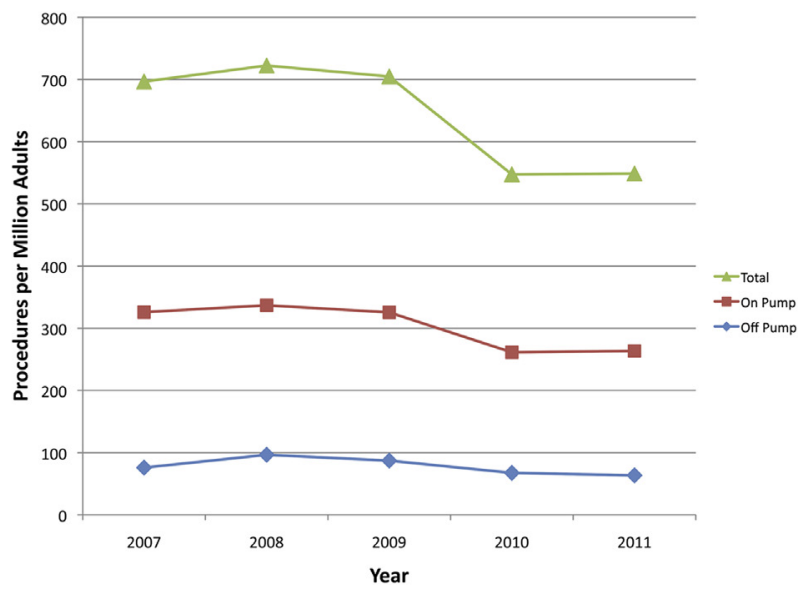

FIGURE 1. CABG rates, 2007 to 2011. A, Elective versus nonelective status ( $P=.16$ for elective; $P=.05$ for nonelective). B, On-pump versus off-pump technique ( $P=.35$ for off-pump; $P=.07$ for on-pump). 


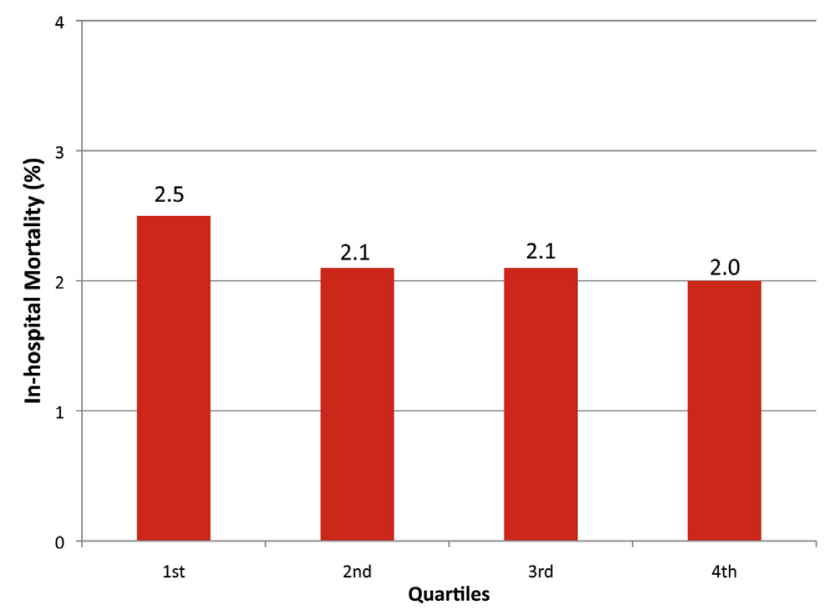

FIGURE 2. In-hospital mortality after isolated CABG $(P=.003)$.

might have impacted patient access to health care and access to coronary procedures/surgeries.

Despite a national reduction in CABG volumes, a previous analysis of NIS data from 1988 to 2003 found a decrease in-hospital adverse events. ${ }^{14}$ Another analysis of the NIS database from 1997 to 2003 demonstrated improving mortality rates after CABG $(3.7 \%$ to $3.3 \%){ }^{18}$ In our analysis, in-hospital mortality after CABG was $2.0 \%$ by 2011 , suggesting that CABG techniques and expertise continue to evolve and adverse events after $\mathrm{CABG}$ are steadily declining in the contemporary era. However, institutions with low CABG volume had an in-hospital mortality of $2.5 \%$, closer to the overall CABG mortality in the year $2003(3.3 \%)$. Although numerous factors can affect patient outcomes after CABG, these data suggest that low hospital CABG volume in current times of decreasing frequency of these operations may be an important contributor to such outcomes and should be considered when evaluating the quality of care associated with CABG. As more institutions have acquired PCI capability in the last decade, the number of CABGcapable institutions has been growing as well, thus diluting the CABG volume and creating more low-volume institutions. It is important to continuously reassess the quality of surgical care in low-volume institutions to ensure that such centers can achieve comparable clinical outcomes to higher-volume centers. In some instances, high-risk patients undergoing complex $\mathrm{CABG}$ procedures may benefit from undergoing these surgeries at higher-volume institutions.

Studies have shown that the quality of surgical care, both perioperatively and postoperatively, continues to evolve for majority of surgical procedures. ${ }^{19}$ Furthermore, recent studies have demonstrated the impact of a learning curve for operators, emphasizing the importance of surgical operator volume..$^{20}$ In fact, the importance of surgical case volume and its effect on the rates of in-hospital mortality with other surgical procedures, such as an open abdominal aortic aneurysm repair, has been clearly shown. ${ }^{21}$ Unfortunately, the declining CABG volume in the last decade may have negatively impacted the training of new

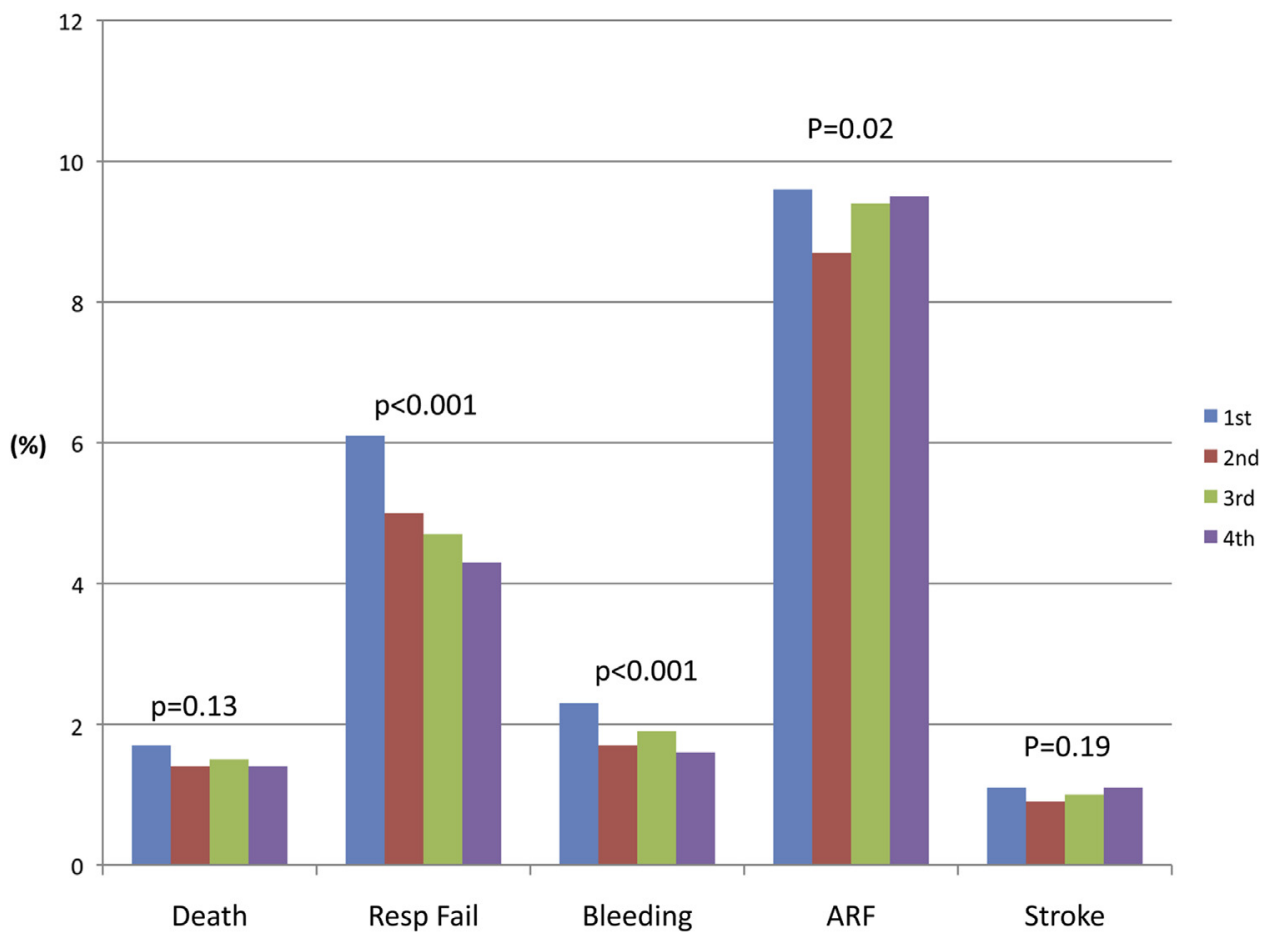

FIGURE 3. Adverse event rates after elective, isolated CABG by quartile of hospital volume. ARF, Acute renal failure. 
TABLE 3. Unadjusted and adjusted associations between hospital volume and outcomes after isolated CABG (with fourth quartile as the reference)

\begin{tabular}{llcccc}
\hline Outcome & Quartile & $\begin{array}{c}\text { Unadjusted OR } \\
(\mathbf{9 5} \% \mathbf{C I})\end{array}$ & $\begin{array}{c}\boldsymbol{P} \\
\text { value }\end{array}$ & $\begin{array}{c}\text { Adjusted OR } \\
(\mathbf{9 5} \% \mathbf{C I})\end{array}$ & $\begin{array}{c}\boldsymbol{P} \\
\text { value }\end{array}$ \\
\hline Death & First & $1.17(1.06-1.29)$ & .002 & $1.39(1.23-1.56)$ & $<.001$ \\
& Second & $1.01(0.94-1.08)$ & .82 & $1.10(1.01-1.20)$ & .02 \\
& Third & $1.03(0.97-1.09)$ & .38 & $1.12(1.04-1.20)$ & .002 \\
\hline
\end{tabular}

This model was adjusted for statistically significant predictor variables that had significant univariate association with outcomes $(P<.05)$ from among left internal mammary artery used, bilateral internal mammary arteries used, off-pump bypass surgery, renal dialysis, previous $\mathrm{CABG}$, previous percutaneous coronary intervention, age, sex, race, hospital bed size, hospital teaching status, region, payer, anemia, collagen vascular disease, congestive heart failure, chronic pulmonary disease, pulmonary circulation disorder, diabetes mellitus, coagulopathy, hypertension, liver disease, neurologic disorders, obesity, peripheral vascular disorders, renal failure, and valvular disease. $O R$, Odds ratio; $C I$, confidence interval.

cardiothoracic surgeons. On the other hand, it is conceivable that further reductions in adverse events after CABG could be achieved over the next decade with improving technology and advances in postoperative care. In fact, a recent analysis of the Netherlands Cardiac Surgery National Database demonstrated that outcomes after valve surgery continue to improve even in the modern era, suggesting that there remains room for evolution in technique and improvement in outcomes. ${ }^{22}$

Previous studies have extensively examined the relationship between CABG volume and associated outcomes after CABG. ${ }^{23,24}$ Rathore et $\mathrm{al}^{25}$ demonstrated an association between low CABG volume $(<250$ cases) and increased perioperative mortality. In our analysis, the median number of CABG cases performed in the quartile with the lowest volume remained unchanged $(\sim 50)$ from 2007 to 2011. At the same time, overall outcomes after CABG have improved over time, and one would have to be careful before establishing a hospital CABG volumebased threshold based on these studies. From a policy standpoint, quality assessment and improvement measures are of great importance for continuously examining and improving CABG outcomes, especially in low-volume centers. Assuming worse outcomes at low-volume centers based solely on retrospective studies may be misleading, however. In fact, a recommendation encouraging the performance of CABG at only high-volume centers would discount low-volume centers with well-established operators demonstrating excellent outcomes after CABG. ${ }^{26}$ Further comprehensive retrospective analyses (eg, from the Society of Thoracic Surgeons database) and randomized clinical studies are needed to make volume threshold recommendations for institutions performing CABG.

\section{Study Limitations}

Several limitations of this study should be acknowledged. First, this is a retrospective study based on data from the NIS, with the sample designed to approximate the national distribution of key hospital characteristics. Our estimates for $\mathrm{CABG}$ were derived from a $20 \%$ sample of US hospitals, and it is possible that CABG volume was either underrepresented or overrepresented by the sample. The NIS does not allow for an analysis of the migration of institutions between quartiles of volume from one year to another; however, the NIS has been used extensively to examine national health care trends, and its sampling design has been validated in numerous publications. Second, unmeasured confounders could not be accounted for despite our best efforts to comprehensively adjust for multiple available clinical variables. The NIS does not include detailed information about patient clinical characteristics, such as frailty, coronary anatomy, angina class, heart failure class, left ventricular function, medications, and smoking status. The absence of these variables complicates comparisons of CABG outcomes by hospital volume, and thus a more detailed exploration of the drivers of the outcomes we observed is beyond the scope of this analysis and the information available in the NIS database. Furthermore, ICD-9-CM codes fail to distinguish between certain preoperative and postoperative complications (eg, respiratory failure, acute respiratory failure, bleeding, stroke); therefore, we analyzed these endpoints only for the elective CABG population. Nonetheless, the hard outcomes of in-hospital mortality should be very reliable in terms of examining the association between CABG mortality and institutional volume. Third, the NIS database provides only in-hospital outcomes, and our findings might not necessarily reflect

TABLE 4. Associations between hospital volume and outcomes after elective, isolated CABG (with fourth quartile as the reference)

\begin{tabular}{|c|c|c|c|c|c|c|}
\hline \multirow[b]{2}{*}{ Outcome } & \multicolumn{2}{|l|}{ First quartile } & \multicolumn{2}{|c|}{ Second quartile } & \multicolumn{2}{|c|}{ Third quartile } \\
\hline & Adjusted OR (95\% CI) & $P$ value & Adjusted OR (95\% CI) & $P$ value & Adjusted OR $(95 \%$ CI $)$ & $P$ value \\
\hline Death & $1.44(1.20-1.74)$ & $<.001$ & $1.05(0.90-1.23)$ & .52 & $1.21(1.08-1.36)$ & .001 \\
\hline Respiratory failure & $1.40(1.24-1.59)$ & $<.001$ & $1.34(1.22-1.46)$ & $<.001$ & $1.11(1.03-1.20)$ & .009 \\
\hline Bleeding & $1.35(1.11-1.64)$ & .002 & $1.05(0.91-1.22)$ & .52 & $1.21(1.08-1.35)$ & .001 \\
\hline Stroke & $0.96(0.75-1.24)$ & .78 & $0.82(0.69-0.98)$ & .03 & $0.88(0.76-1.02)$ & .08 \\
\hline Acute renal failure & $1.10(0.99-1.22)$ & .08 & $1.00(0.93-1.08)$ & .99 & $1.10(1.04-1.17)$ & .001 \\
\hline Shock & $1.03(0.86-1.23)$ & .75 & $0.95(0.84-1.07)$ & .41 & $1.21(1.12-1.33)$ & $<.001$ \\
\hline
\end{tabular}

This model was adjusted for the statistically significant predictor variables used in Table 3. OR, Odds ratio; $C I$, confidence interval. 
TABLE 5. Independent predictors of in-hospital mortality after isolated CABG

\begin{tabular}{lcr}
\hline \multicolumn{1}{c}{ Variables } & Adjusted OR $(\mathbf{9 5} \%$ CI) & $\boldsymbol{P}$ value \\
\hline Congestive heart failure & $3.52(2.97-4.15)$ & $<.001$ \\
Chronic renal failure & $2.03(1.87-2.20)$ & $<.001$ \\
Liver disease & $2.07(1.63-2.62)$ & $<.001$ \\
Previous CABG & $1.51(1.18-1.95)$ & .001 \\
Peripheral vascular disease & $1.52(1.40-1.64)$ & $<.001$ \\
Female sex & $1.45(1.35-1.56)$ & $<.001$ \\
Low hospital volume & $1.34(1.17-1.52)$ & $<.001$ \\
\hline
\end{tabular}

$O R$, Odds ratio; $C I$, confidence interval; $C A B G$, coronary artery bypass grafting.

long-term outcomes after $\mathrm{CABG}$, commonly examined in randomized trials; however, given that most adverse outcomes after $\mathrm{CABG}$ occur soon after the procedure, this analysis provides important insights regarding perioperative outcomes in relation to $\mathrm{CABG}$ volume. Finally, the inability to incorporate operator volume limits the application of our analysis in terms of analyzing the outcomes for high-volume surgeons in low-volume centers. Further studies are needed to assess the relationship between operator CABG volume and outcomes.

\section{CONCLUSIONS}

The rate of CABG surgery has declined steadily from 2007 to 2011, driven mainly by a reduction in CABG utilization in higher-volume centers. Elective CABG in low-volume centers was associated with a higher frequency of adverse in-hospital events. Undergoing isolated CABG at low-volume institutions was an independent predictor of in-hospital all-cause mortality. Institutional quality assessment and improvement measures are needed to continuously examine and improve CABG outcomes, particularly in low-volume centers.

\section{Conflict of Interest Statement}

D.N.F. has received consulting/speaker's fees from Eli Lilly, Daiichi-Sankyo, Abbott Vascular, Pfizer, and Bristol-Myers Squibb. All other authors have nothing to disclose with regard to commercial support.

\section{References}

1. AbuRahma AF, Stone PA, Srivastava M, Hass SM, Mousa AY, Dean LS, et al. The effect of surgeon's specialty and volume on the perioperative outcome of carotid endarterectomy. J Vasc Surg. 2013;58:666-72.

2. Pettit SJ, Jhund PS, Hawkins NM, Gardner RS, Haj-Yahia S, McMurray JJ, et al. How small is too small? A systematic review of center volume and outcome after cardiac transplantation. Circ Cardiovasc Qual Outcomes. 2012;5:783-90.

3. Chikwe J, Cavallaro P, Itagaki S, Seigerman M, Diluozzo G, Adams DH. National outcomes in acute aortic dissection: influence of surgeon and institutional volume on operative mortality. Ann Thorac Surg. 2013;95:1563-9.

4. Strom JB, Wimmer NJ, Wasfy JH, Kennedy K, Yeh RW. Association between operator procedure volume and patient outcomes in percutaneous coronary intervention: a systematic review and meta-analysis. Circ Cardiovasc Qual Outcomes. 2014;7:560-6.
5. Ch'ng SL, Cochrane AD, Wolfe R, Reid C, Smith CI, Smith JA. Procedure-specific cardiac surgeon volume associated with patient outcome following valve surgery, but not isolated CABG surgery. Heart Lung Circ. 2015;24:583-9.

6. Lapar DJ, Mery CM, Kozower BD, Kern JA, Kron IL, Stukenborg GJ, et al. The effect of surgeon volume on mortality for off-pump coronary artery bypass grafting. J Thorac Cardiovasc Surg. 2012;143:854-63.

7. Post PN, Kuijpers M, Ebels T, Zijlstra F. The relation between volume and outcome of coronary interventions: a systematic review and meta-analysis. Eur Heart J. 2010;31:1985-92.

8. Hockenberry JM, Lien HM, Chou SY. Surgeon and hospital volume as quality indicators for CABG in Taiwan: examining hazard to mortality and accounting for unobserved heterogeneity. Health Serv Res. 2010;45(5 Pt 1):1168-87.

9. Sakata R, Kuwano H, Yokomise H. Hospital volume and outcomes of cardiothoracic surgery in Japan: 2005-2009 national survey. Gen Thorac Cardiovasc Surg. 2012;60:625-38.

10. Healthcare Cost and Utilization Project (HCUP). Overview of the National (Nationwide) Inpatient Sample (NIS), 2007-2009. Available at: http://www. hcup-us.ahrq.gov/nisoverview.jsp. Accessed December 1, 2014.

11. Mohan AV, Fazel R, Huang PH, Shen YC, Howard D. Changes in geographic variation in the use of percutaneous coronary intervention for stable ischemic heart disease after publication of the Clinical Outcomes Utilizing Revascularization and Aggressive Drug Evaluation (COURAGE) trial. Circ Cardiovasc Qual Outcomes. 2014;7:125-30.

12. Elixhauser A, Steiner C, Harris DR, Coffey RM. Comorbidity measures for use with administrative data. Med Care. 1998;36:8-27.

13. US Census Bureau. 2007-2011 ACS 5-year Public Use Microdata Samples (PUMS). Available at: http://www2.census.gov/acs2011_5yr/pums/csv_pus. zip. Accessed October 31, 2014.

14. Ricciardi R, Virnig BA, Ogilvie JW Jr, Dahlberg PS, Selker HP, Baxter NN Volume-outcome relationship for coronary artery bypass grafting in an era of decreasing volume. Arch Surg. 2008;143:338-44.

15. Epstein AJ, Polsky D, Yang F, Yang L, Groeneveld PW. Coronary revascularization trends in the United States, 2001-2008. JAMA. 2011;305: 1769-76.

16. Ford ES, Ajani UA, Croft JB, Critchley JA, Labarthe DR, Kottke TE, et al. Explaining the decrease in US deaths from coronary disease, 1980-2000. N Engl J Med. 2007;356:2388-98.

17. Ryan J, Linde-Zwirble W, Engelhart L, Cooper L, Cohen DJ. Temporal changes in coronary revascularization procedures, outcomes, and costs in the bare-metal stent and drug-eluting stent eras: results from the US Medicare program. Circulation. 2009;119:952-61.

18. LaPar DJ, Stukenborg GJ, Guyer RA, Stone ML, Bhamidipati CM, Lau CL, et al. Primary payer status is associated with mortality and resource utilization for coronary artery bypass grafting. Circulation. 2012;126(11 Suppl 1):S132-9.

19. Ettema RG, Van Koeven H, Peelen LM, Kalkman CJ, Schuurmans MJ Preadmission interventions to prevent postoperative complications in older cardiac surgery patients: a systematic review. Int J Nurs Stud. 2014;51:251-60.

20. Maruthappu M, Duclos A, Lipsitz SR, Orgill D, Carty MJ. Surgical learning curves and operative efficiency: a cross-specialty observational study. BMJ Open. 2015;5:e006679.

21. McPhee JT, Robinson WP III, Eslami MH, Arous EJ, Messina LM, Schanzer A. Surgeon case volume, not institution case volume, is the primary determinant of in-hospital mortality after elective open abdominal aortic aneurysm repair. $J$ Vasc Surg. 2011;53:591-9.e2.

22. Siregar S, de Heer F, Groenwold RH, Versteegh MI, Bekkars JA, Brinkman ES, et al. Trends and outcomes of valve surgery: 16-year results of the Netherlands Cardiac Surgery National Database. Eur J Cardiothorac Surg. 2014;46:386-97.

23. Lin HC, Xirasagar S, Tsao NW, Hwang YT, Kuo NW, Lee HC. Volume-outcome relationships in coronary artery bypass graft surgery patients: 5-year major cardiovascular event outcomes. J Thorac Cardiovasc Surg. 2008;135:923-30.

24. Peterson ED, Coombs LP, DeLong ER, Haan CK, Ferguson TB. Procedural volume as a marker of quality for CABG surgery. JAMA. 2004;291:195-201.

25. Rathore SS, Epstein AJ, Volpp KG, Krumholz HM. Hospital coronary artery bypass graft surgery volume and patient mortality, 1998-2000. Ann Surg. 2004; 239:110-7.

26. Early GL, Roberts SR. Excellence and low case volume: an example of the inapplicability of volume-based credentialing. Ann Thorac Surg. 2000;69:146-50.

Key Words: coronary artery bypass grafting, volume, outcomes 